\title{
Comparison of bus driving cycles elaborated for vehicle dynamic simulation
}

\author{
Attila Vámosi $^{1 *} \odot$, Levente Czégé ${ }^{2}$ and Imre Kocsis ${ }^{1}$ \\ ${ }^{1}$ Department of Basic Technical Studies, Faculty of Engineering, University of Debrecen, Debrecen, \\ Hungary \\ ${ }^{2}$ Department of Mechanical Engineering, Faculty of Engineering, University of Debrecen, Debrecen, \\ Hungary
}

International Review of Applied Sciences and Engineering

12 (2021) 1, 86-91

DOl:

$10.1556 / 1848.2020 .00153$

(c) 2020 The Author(s)

Received: July 12, 2020 • Accepted: December 14, 2020

Published online: January 7, 2021

\section{ORIGINAL RESEARCH PAPER}

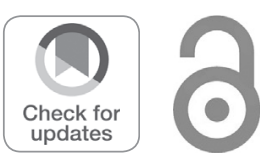

\begin{abstract}
Due to the technological progress, new approaches such as model-based design are spreading in the development process in the automotive industry to meet the increased requirements related to lower fuel consumption and reduced emission. This work is part of a research project which focuses on dynamic modeling of vehicles aimed at analyzing and optimizing the emission and fuel consumption. To model the driver behavior, the simulation control algorithm requires a predetermined speed-time curve as an input. The completeness of this driving cycle is a crucial factor in the simulation, and as far as the legislative driving cycles are not accurate enough, it is indispensable to develop our own one representing our narrower area and driving conditions. This article considers two common drive cycle design methods, comparing the micro-trip-based approach and the Markov-chain approach. The new driving cycle has been developed applying the Markov-chain approach and compared to a driving cycle introduced in our recent paper using the micro-trip method. The comparison basis is the Speed-Acceleration Probability Distribution, which sufficiently reflects the dynamic behavior of the vehicle, and the root mean square error, including parameters such as the average speed, average cruising speed, average acceleration, average deceleration, root mean square acceleration, and idle time percentage. The representative Bus Driving Cycle for Debrecen is prepared to be applied in the vehicle dynamics simulation for evaluating and improving the fuel economy of vehicles, selecting the proper power source for various applications and the optimization of the powertrain and the energy consumption in researches to be continued.
\end{abstract}

\section{KEYWORDS}

driving cycle, real traffic data, urban bus route, Markov chain, micro-trip method

\section{INTRODUCTION}

As a result of the increasingly stringent measures of the governments as well as the market requirements large efforts are made to reduce fuel consumption and emission in the automotive industry. At the same time, due to technological progress new methods and tools are involved in the development process such as computer modeling and simulation. At the University of Debrecen, the project has been launched with emphasis on vehicle dynamics simulation. Among the goals we find the optimization of fuel consumption of various vehicle design concepts of propulsion system, such as internal combustion engine, fuel cell, electric drive, and hybrid systems. The basis of the analysis and optimization is a dynamic model which consists of a simplified vehicle model and discrete control algorithms to execute the desired driving cycle inputs for the vehicle. The math model representing the vehicle mechanics is a simplified longitudinal "bicycle" model including the power loss effects like drag and rolling resistance, the drivetrain, the brake system and the model of the power source (internal combustion engine or electric motor). The control algorithm is based on PID controllers to model the driver behavior. 
The input of the PID controllers is the speed of the vehicle, which is given by a predetermined time-speed curve. This is the so-called driving cycle, which represents the driving pattern of the analyzed vehicle. The level of the representativeness of the driving cycle is a crucial factor in the vehicle dynamics simulation, therefore it is an important task to find the appropriate method of data collection, data processing, and driving cycle development. The existing methods are fundamentally based on the analysis of large amounts of long-term data collected under real-world conditions and apply some statistical procedures to develop a representative driving cycle of the desired length. It has also been proven in several papers that the legislative driving cycles are not accurate enough to estimate the fuel and energy consumption or emission data for vehicles, because the driving patterns can be very different in various areas and cities [1]. The conclusion that can be drawn from these studies is that the development of alternative driving cycle characteristics of driving conditions in our area is necessary to get accurate results from the dynamic vehicle simulation.

In this paper two driving cycles for Debrecen are overviewed and compared. They were obtained by the two common driving cycle development techniques, namely the micro-trip-based approach and the Markov-chain approach. In Vámosi et al. [2] a driving cycle is introduced constructed with the micro-trip method for city buses in Debrecen, Hungary. Now a new driving cycle is presented developed with the Markov-chain approach using the same dataset. Finally, the two approaches are being compared based on the root mean square error and the Speed-Acceleration Probability Distribution (SAPD), which precisely reflects the dynamic behavior of the vehicle.

\section{LITERATURE REVIEW}

The different versions of the micro-trip-based approach have several advantages. But the parameters used for microtrip-based cycles are not directly related to emissions, while applications aiming at emission reduction of vehicles need emission sensitive driving characteristics, as it is emphasized in several publications, see e.g., Silvas [3] or Delgado-Neira [4].

The Markov modal events studied in Lin and Niemeier [5] demonstrate the stochastic nature of the driving data. In practice it is a significant contribution to the estimation of emissions that this model can represent the modal events and driving changeableness, while modal operating conditions are not significantly distinguished by the micro-trip approach [5]. The Markov process allows us to replicate the global driving characteristics while preserving small timescale speed fluctuations that contain the information related to driving variability [6].

The Markov process theory was applied to depict how real driving occurs according to a transition probability matrix by Lin and Niemeier [5]. Dai et al. [7] got a more sophisticated approach modifying the Markov process. They applied several variables related to emissions, matching with the modal distributions and SAPD, furthermore, a complex parameter for assessment was used to allow the driving mode to fit small accelerations while considering significant acceleration rates to be classified as acceleration modes, respectively.

The micro-trip-based and Markov theory-based methods were compared and the limitations of both methods were presented in many papers. According to Dai et al. [7] the feature that the micro-trip approach does not make difference between various types of driving conditions could be considered as a limitation. If, for example, a vehicle does not have to stop frequently due to smooth traffic conditions, several diverse road segments or traffic conditions could be included in a single micro-trip. Traffic signals and congestion generate most of the stop-go driving patterns and lead to higher fuel consumption, since more fuel is consumed by vehicles during stop-go conditions [8]. Consequently, micro-trip-based methods have been applied primarily in situations where the goal is to develop a driving cycle for a single type of trip or for region-wide driving conditions [7].

Lin and Niemeier [5] compared two frequently referred cycles, namely the LA01 cycle (based on the Markov process theory) and the Unified Cycle (based on micro-trips). Significant differences were obtained related to frequencies, durations and intensities of modal events, though both cycles represent the global characteristics in connection with observed speed and acceleration in the driving dataset. Compared with the Unified Cycle, the LA01 contains modal events with frequencies and durations more similar to those of the sample dataset. The stochastic method deals with a couple of major problems appearing in the construction procedure of the Unified Cycle. In the Unified Cycle the global characteristics is preserved, while small timescale activities, for example small accelerations are lost, in spite of their crucial role in increased emissions. If we use microtrips, within a micro-trip, we cannot distinguish significantly the modal operating conditions. This can result an imprecise representation of the modal events. Considering the frequency, average duration and intensity, generally, LA01 replicates the vehicle operation modes in the sample dataset better than the Unified Cycle. Some limitations of the Markov method are also mentioned. For instance, the segment-to-segment speed match criterion is crucial when transition matrix is used, and the accuracy highly depends on the sample size [5]. The research presented in Shi et al. [9] shows that only under the condition of small time intervals does certain probability state transfer exist between the current state and the next one.

Authors founded a theory for developing driving cycles in Shi et al. [9]. The authors proved that a driving cycle has the Markov property and that the application of transition matrix is an appropriate method of developing driving cycles. Considering that micro-trip-based driving cycles could not fit the dynamometer test, developing driving cycles by following its essential characteristics could be a more appropriate method. 


\section{SELECTION OF THE APPROPRIATE DRIVING CYCLE CONSTRUCTION METHOD}

\subsection{Description of Markov chain method}

Markov chain is a mathematical tool used to model a random process, where the so-called Markov property, which can be expressed by

$$
\begin{aligned}
P\left(X_{n+1}\right. & \left.=x \mid X_{1}=x_{1}, X_{2}=x_{2}, \ldots, X_{n}=x_{n}\right)=P\left(X_{n+1}\right. \\
& \left.=x \mid X_{n}=x_{n}\right),
\end{aligned}
$$

that is, the next state depends on the current state only.

We can write the one-step transition probability from state $x_{i}$ to state $x_{j}$ as

$$
p_{i j}=P\left(X_{n+1}=x_{j} \mid X_{n}=x_{i}\right), \quad i, j=1, \ldots, N,
$$

where $N$ denotes the number of the states. The one-step state probabilities constitute the so-called transition probability matrix.

\subsection{Data processing}

In this method, the states can be characterized by the average speed and the acceleration values calculated from the speed change measured at the same time interval. Since the instantaneous speed value was read every second during the measurement, the average speed shall be determined by the following formula

$$
\bar{v}_{i}=\frac{v_{i}+v_{i-1}}{2} .
$$

Acceleration values are also available from the measurement, but they are also instantaneous values, and the values calculated from the actual speed change are required for the calculation. The speed is measured in $\mathrm{km} / \mathrm{h}$ unit and the time interval is $1 \mathrm{~s}$, so the acceleration value can be calculated with this formula

$$
\bar{a}_{i}=\frac{v_{i}-v_{i-1}}{3.6} .
$$

The data were discretized in step of $1 \mathrm{~km} / \mathrm{h}$ in speed and $0.2 \mathrm{~m} / \mathrm{s}^{2}$ in acceleration following the method used e.g., in Torp and Önnegren [10]. Figure 1 shows the speed-acceleration distribution of discretized points gained from the full dataset.

\subsection{Calculation of the transition probability matrix}

To determine the number of states we have to consider the measured maximum speed and absolute maximum acceleration values and have to fix the resolution of speed and acceleration. In our investigations the resolutions followed from the discretization steps $a_{\text {res }}=0.2 \mathrm{~m} / \mathrm{s}^{2}$ and $v_{\text {res }}=1$ $\mathrm{km} / \mathrm{h}$, respectively.

We checked many pairs and $\left(a_{\text {res }}, v_{\text {res }}\right)=\left(0.2 \mathrm{~m} / \mathrm{s}^{2}, 1 \mathrm{~km} / \mathrm{h}\right)$ was found to be the most appropriate one from the point of view of the accuracy of the final driving cycle. Although the application of larger discretization steps (coarser resolution) results a smaller matrix, and consequently shorter computation time, the accuracy of the result is much worse. This resolution is used in several literatures as well. The number of the rows and columns were calculated by equations

$$
n_{r}=2 \cdot \frac{\left|a_{\max }\right|}{a_{\text {res }}}+1 ; \quad n_{c}=\frac{v_{\max }}{v_{\text {res }}}+1 .
$$

Each speed-acceleration pair represents a state, so we have $n_{r} \cdot n_{c}$ different states.

\subsection{Generating the candidate driving cycles}

The duration of the desired driving cycle is determined by the needs of the simulation. According to Huang et al. [1] the length of the representative driving cycle should be between 500 and $3,000 \mathrm{~s}$. Based on the requirements of the simulation and the recommendations in the literature, we used 1,200 s as the length of the driving cycle.

The driving cycle has to start with the idle state where the speed and the acceleration are zero. The transition probability matrix is used to determine the next state. A random number $r$ is generated in interval $(0,1)$, and if $r$ satisfies the inequality

$$
\sum_{j=1}^{k-1} p_{i j}<r \leq \sum_{j=1}^{k} p_{i j} \quad(1 \leq k \leq N),
$$

then the next state is set to state $k$.

In the new state the speed and acceleration are given by the discretized dataset. Repeat this method until the duration of the driving cycle reaches or leaves the desired length and the actual state is the idle state. We generated 10 candidate driving cycles using this method.

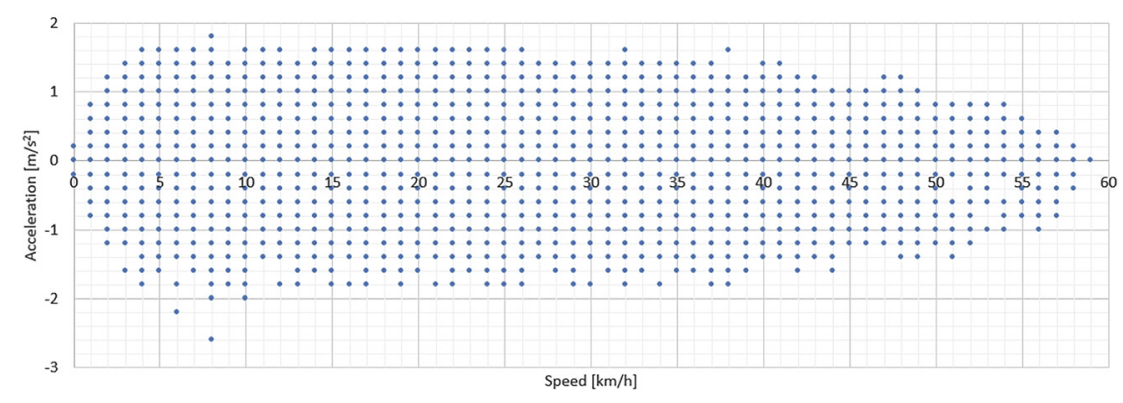

Fig. 1. Discretized dataset 


\section{RESULTS}

\subsection{Selection and analysis of representative driving cycle}

To validate each generated driving cycle the following variables were used:

- Average speed $\left(v_{\text {total }}\right.$ in $\left.\mathrm{km} / \mathrm{h}\right)$ : Average value of the speed during a driving segment.

- Average cruising speed $\left(v_{\text {cruise }}\right.$ in $\left.\mathrm{km} / \mathrm{h}\right)$ : Average value of the non-zero speeds.

- Average acceleration $\left(a\right.$ in $\left.\mathrm{m} / \mathrm{s}^{2}\right)$ : Average value of the positive accelerations.

- Average deceleration $\left(d\right.$ in $\left.\mathrm{m} / \mathrm{s}^{2}\right)$ : Average value of the negative accelerations.

- Root mean square acceleration $\left(a_{R M S}\right.$ in $\left.\mathrm{m} / \mathrm{s}^{2}\right)$ : root mean square of the positive and negative accelerations, calculated as

$$
a_{R M S}=\sqrt{\sum_{i=1}^{n}\left(\frac{x_{i}-\bar{x}_{i}}{\bar{x}_{i}}\right)^{2}} .
$$

- Idle time percentage ( $t_{i d l e}$ in \%): the quotient of the idle time and the total time in percentage.

To select the most representative driving cycle we used the root mean square (RMS) error. All of the parameters were calculated for each candidate driving cycle and were compared to the parameters of the full dataset. Table 1 shows the characteristic parameters of the candidate driving cycles.

An alternative tool to describe driving patterns is based on the Speed-Acceleration Probability Distribution (SAPD) [11]. This method classifies the average speed and average acceleration of the vehicles into speed-acceleration bins. The similarity between the SAPD of a candidate driving cycle and the SAPD of the full dataset is another useful indicator of the representativeness. We used the Quality of Fit (QoF) value

$$
Q o F=\sum_{i=1}^{n} \sum_{j=1}^{m}\left(P_{i j, \text { candidate }}-P_{i j, f u l l d a t a s e t}\right)^{2}
$$

to evaluate the degree of similarity between SAPDs, where $P_{i j}$ is the probability that the vehicle travels within the bin $i$ of speed and the bin $j$ of acceleration, $n$ is the total number of the bins of speed, and $m$ is the total number of the bins of acceleration. To define the number of bins we used $v_{i n t}=$ $5 \mathrm{~km} / \mathrm{h}$ and $a_{\text {int }}=0.5 \mathrm{~m} / \mathrm{s}^{2}$ as the length of speed and acceleration intervals, respectively.

As Table 1 shows, cycles DC6 and DC7 have the lowest RMS value. The QoF value of DC7 is 0.008 , while that of DC6 is only 0.002 , which indicates very good accuracy, that is, this driving cycle represents better the full dataset than the others. Figure 2 shows the constructed driving cycle as a speed-time chart. It has total time of $1,208 \mathrm{~s}$.

\subsection{Comparison with driving cycle based on the micro-trip method}

In Vámosi et al. [2] a Bus Driving Cycle was constructed for Debrecen using the micro-trip method. There the total time of the cycle was $747 \mathrm{~s}$, the further parameters of the driving cycle are collected in Table 2.

As we can see from the results in Table 2, both driving cycles have similar RMS error, but the driving cycle constructed with the Markov chain method has lower QoF value than the driving cycle constructed with the microtrip method. Figure 3 shows the SAPD of the driving pattern and the SAPDs of the two driving cycles obtained with the micro-trip method and the Markov chain method, respectively. It can be seen that both SAPDs look similar to the SAPD of the driving pattern, but level of similarity is higher in the case of the Markov chain method.

\begin{tabular}{|c|c|c|c|c|c|c|c|c|c|c|c|}
\hline Parameter & Full dataset & DC1 & DC2 & DC3 & DC4 & DC5 & DC6 & DC7 & DC8 & DC9 & DC10 \\
\hline$v_{\text {total }}(\mathrm{km} / \mathrm{h})$ & 13.837 & 11.705 & 15.172 & 15.542 & 13.810 & 10.478 & 12.793 & 14.034 & 8.943 & 16.268 & 15.634 \\
\hline$v_{\text {cruise }}(\mathrm{km} / \mathrm{h})$ & 22.659 & 20.925 & 22.561 & 24.334 & 21.177 & 20.385 & 21.754 & 22.764 & 20.521 & 23.844 & 22.891 \\
\hline$a\left(\mathrm{~m} / \mathrm{s}^{2}\right)$ & 0.773 & 0.762 & 0.828 & 0.768 & 0.698 & 0.760 & 0.817 & 0.718 & 0.797 & 0.804 & 0.794 \\
\hline$d\left(\mathrm{~m} / \mathrm{s}^{2}\right)$ & -0.648 & -0.641 & -0.659 & -0.656 & -0.592 & -0.623 & -0.651 & -0.668 & -0.615 & -0.660 & -0.556 \\
\hline$a_{R M S}\left(\mathrm{~m} / \mathrm{s}^{2}\right)$ & 0.602 & 0.566 & 0.647 & 0.621 & 0.573 & 0.539 & 0.613 & 0.574 & 0.497 & 0.665 & 0.592 \\
\hline$t_{\text {idle }}(\%)$ & 38.935 & 44.059 & 32.750 & 36.130 & 34.786 & 48.598 & 41.191 & 38.350 & 56.421 & 31.776 & 31.703 \\
\hline RMS error & & 0.225 & 0.214 & 0.165 & 0.186 & 0.378 & 0.120 & 0.092 & 0.608 & 0.284 & 0.270 \\
\hline
\end{tabular}

Table 1. Characteristic parameters of the candidate driving cycles

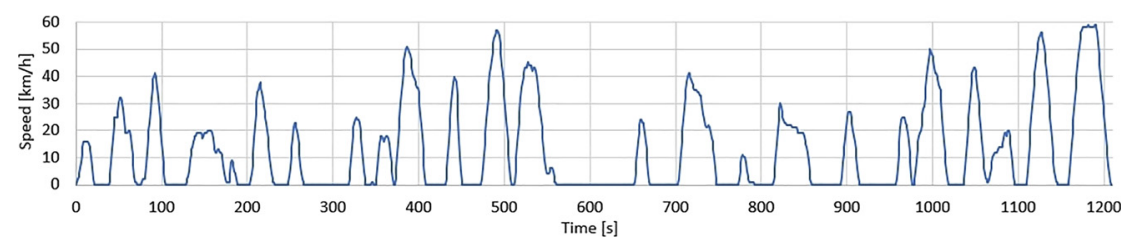

Fig. 2. Proposed driving cycle 
Table 2. Driving cycle characteristic parameters

\begin{tabular}{|c|c|c|c|}
\hline Parameter & Full dataset & Micro-trip method & Markov chain method \\
\hline Total time (s) & 15,658 & 747 & 1,208 \\
\hline Cruise time (s) & 9,561 & 450 & 710 \\
\hline Idle time $(\mathrm{s})$ & 6,097 & 297 & 498 \\
\hline Idle time percentage (\%) & 38.939 & 36.142 & 41.191 \\
\hline Average speed $(\mathrm{km} / \mathrm{h})$ & 13.837 & 13.025 & 12.793 \\
\hline Average cruise speed $(\mathrm{km} / \mathrm{h})$ & 22.659 & 19.197 & 21.754 \\
\hline Maximum speed $(\mathrm{km} / \mathrm{h})$ & 59.0 & 56.8 & 59.0 \\
\hline Average acceleration $\left(\mathrm{m} / \mathrm{s}^{2}\right)$ & 0.773 & 0.295 & 0.817 \\
\hline Average deceleration $\left(\mathrm{m} / \mathrm{s}^{2}\right)$ & -0.648 & -0.314 & -0.651 \\
\hline Root mean square acceleration $\left(\mathrm{m} / \mathrm{s}^{2}\right)$ & 0.602 & 0.571 & 0.613 \\
\hline Maximum acceleration $\left(\mathrm{m} / \mathrm{s}^{2}\right)$ & 1.8 & 1.57 & 1.6 \\
\hline Maximum deceleration $\left(\mathrm{m} / \mathrm{s}^{2}\right)$ & -2.8 & -1.62 & -2.4 \\
\hline RMS error & & 0.082 & 0.122 \\
\hline Quality of Fit & & 0.028 & 0.002 \\
\hline
\end{tabular}

a)

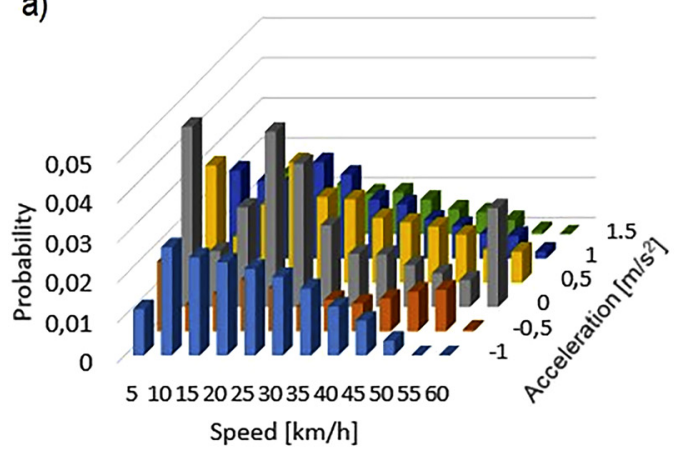

b)

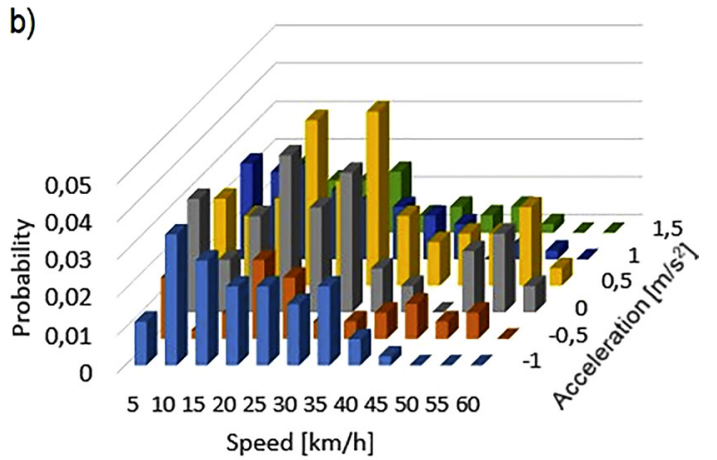

c)

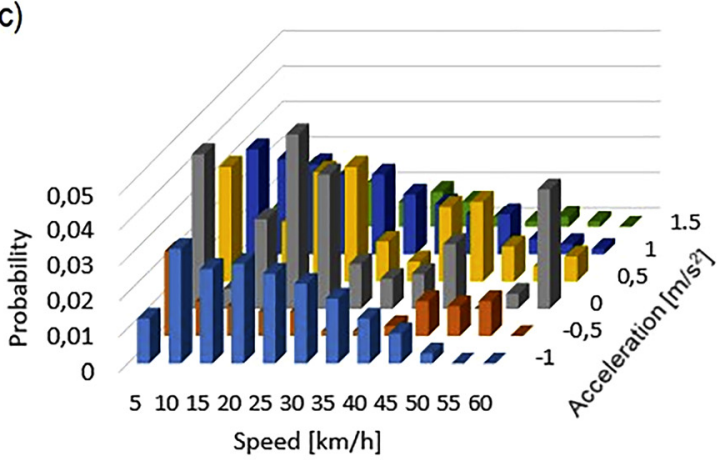

Fig. 3. Speed-Acceleration Probability Distributions (a) full dataset, (b) micro-trip method, (c) Markov chain method

\section{CONCLUSIONS}

In this paper we compared two Bus Driving Cycles for Debrecen constructed with two common techniques of driving cycle development, namely the micro-trip-based approach and the Markov-chain approach. We introduced the application of Markov chain approach, then we compared it to the driving cycle developed using the microtrip method focusing on the requirements of a dynamic simulation, which is a crucial tool of research project aiming at the analysis and optimization of emission and fuel consumption of various vehicles. As a result of our research it can be stated that both driving cycles have similar RMS error values based on certain kinematic parameters, but the driving cycle developed with the help of the Markov chain method shows better correspondence to the original dataset on the basis of the SAPD, thus it has better relation to energy consumption. This driving cycle has a time duration of 1,208 $s$ and an average speed of $12.793 \mathrm{~km} / \mathrm{h}$, the degree of similarity between SAPDs, in terms of the Quality of Fit value, is 0.002 , which means very good accuracy. The new representative Bus Driving Cycle for Debrecen will be applied in a dynamic vehicle simulation-based development process aiming at the improvement of fuel economy of vehicles, the 
proper selection of the power source for various applications and the optimization of the powertrain and energy consumption.

\section{ACKNOWLEDGMENT}

The research was financed by the Thematic Excellence Programme of the Ministry for Innovation and Technology in Hungary (ED_18-1-2019-0028), within the framework of the (Automotive Industry) thematic programme of the University of Debrecen.

\section{REFERENCES}

[1] W. T. Huang, H.Y. Tong, C. P. Lee, K. Ha, and L.Y. Pao, "Development of a practical driving cycle construction methodology: A case study in Hong Kong," Transp. Res. D Transp. Environ., vol. 12, no. 2, pp. 115-28, 2007, https://doi.org/10.1016/j.trd.2007.01.002.

[2] A. Vámosi, L. Czégé, and I. Kocsis, Development of Bus Driving Cycle for Debrecen on the Basis of Real-traffic Data. Periodica Polytechnica Transportation, 2020, (accepted, in press).

[3] E. Silvas, Integrated Optimal Design for Hybrid Electric Vehicles. Eindhoven: Doctor of Philosophy, Department of Mechanical Engineering, 2015. https://research.tue.nl/files/8809090/ 20151130_Silvas.pdf.

[4] O. F. Delgado-Neira, Driving Cycle Properties and their Influence on Fuel Consumption and Emissions. Graduate Theses, Dissertations, and Problem Reports. 3568, 2012. https://researchrepository.wvu. edu/etd/3568.

[5] J. Lin and D. Niemeier, "An exploratory analysis comparing a stochastic driving cycle to California's regulatory cycle," Atmos. Environ., vol. 36, pp. 5759-70, 2002, https://doi.org/10.1016/ S1352-2310(02)00695-7.

[6] Y. Li, J. Peng, H. He, and S. Xie, "The study on multi-scale prediction of future driving cycle based on Markov chain," Energy Procedia, vol. 105, pp. 3219-24, 2017, https://doi.org/10.1016/j. egypro.2017.03.709.

[7] Z. Dai, D. Niemeier, and D. Eisinger, Driving Cycles: A New Cyclebuilding Method that Better Represents Real-world Emissions. 2008. https://www.academia.edu/26820568.

[8] U. Galgamuwa, L. Perera, and S. Bandara, "Developing a general methodology for driving cycle construction: comparison of various established driving cycles in the world to propose a general approach," J. Transport. Technol., vol. 5, pp. 191-203, 2015, https://doi.org/10.4236/jtts.2015.54018.

[9] S. Shi, N. Lin, Y. Zhang, J. Cheng, and C. Huang, "Research on Markov property analysis of driving cycles and its application," Transport. Res. D, vol. 47, pp. 171-81, 2016, https://doi.org/10. 1016/j.trd.2016.05.013.

[10] E. Torp and P. Önnegren, Driving Cycle Generation Using Statistical Analysis and Markov Chains. Dissertation, 2013. Retrieved from http://urn.kb.se/resolve?urn=urn:nbn:se:liu:diva-94147.

[11] J. I. Huertas, L. F. Quirama, M. D. Giraldo, and J. Díaz, "Comparison of driving cycles obtained by the Micro-trips, Markovchains and MWD-CP methods," Int. J. Sustain. Energy Plann. Manage., vol. 22, pp. 109-20, 2019, https://doi.org/10.5278/ijsepm. 2554. 